\title{
Verificación de Relaciones Entre Pruebas Neurológicas y Rendimiento Escolar
} \author{
Drs. Alejandro Marterola A. ${ }^{1}$, Alfredo Avendaño B. ${ }^{2}$, Carlos Valenzuela Y. ${ }^{2}$, Irene Morales $\mathrm{C}^{3}$,
Alex Colombo L. , Gustavo Castillo O. ${ }^{4}$, y Raúl Araya R.

\section{Verification of The Relationships Between Neurological} \\ and Reading-Writing Performances
}

\begin{abstract}
In previous studies we mentioned some neurological tests that could discriminate children in groups of different performances in the reading-writing process. These studies included several bias of ascertaimment, this being the reason which decided us to perform the present research. Discriminant, doubtful discriminant and mon discriminant tests were included. The discriminant power of each test was, in general, confirmed. The sex dimorphism in the reading-writing performance found in previous studies was also found in this research but it was not significant.
\end{abstract}

Correlacionar los resultados de algunas pruebas de técnica sencilla, pero capaces de revelar funciones neuropsicológicas completamente integradas, con la capacidad de aprendizaje de la lecto-escritura en los primeros 5 semestres de asistencia escolar continua, fue uno de los aspectos estudiados en una cohorte de nifios seguidos longitudinalmente ${ }^{1 \cdot 2 \cdot 3}$.

Los resultados mostraron correlaciones significativas, pero, los sesgos inherentes a este tipo de estudio poblacional nos hicieron plantear la conveniencia de hacer contrapruebas que satisficieran las exigencias de una metodología más depurada. La necesidad de ofrecer conclusiones más definitivas en esta importante cuestión es el objetivo de la investigación complementaria que se presenta ahora.

De los resultadios más notorios de la experiencia longitudinal cabe mencionar: a) Los varones presentan un desarrollo de maduración escolar $y$

1 Departamento de Pediatría. Fac. de Medicina. Universidad de Chile.

2 Departamento de Biol. Celular y Genética. Fac. de Medicina. U. Chile.

3 CECOM.

4 Alumnos Internos Fac. de Medicina. neurológico retrasado en relación a las mujeres en las edades examinadas ( -10 años); b) Hay escaso número de mujeres clasificadas como de rendimiento subnormal; c) Las pruebas neurológicas que significan la mayor complejidad de las funciones sensorio-motoras y de integración cortical resultaron correlacionadas con rendimiento escolar en lecto-escritura, así por ejemplo la prueba que consiste en la imitación por parte del niño de una configuración digital que el examinador le muestra, resultó muy discriminatoria en cuanto a la capacidad de lecto-escritura; d) En cambio, las pruebas relacionadas con actividades reflejas simples no resultaron discriminativas; e) Pudimos generar predicciones bayesianas de probabilidades de rendimiento escolar dado que se tenía un determinado rendimiento en una prueba neuroló. gica 4 .

Como no estimamos que estos resultados eran lo suficientemente concluyentes, decidimos realizar su verificación siguiendo la metodología científica ordinaria con una probabilidad "a priori" estimada por la inversión bayesiana que sirve de hipótesis para ser verificada en un experimento ulterior. En este caso nos interesa verificar si las pruebas que resultaron discriminativas y las que no lo fueron repiten sus cualidades en un experimento 
diferente, en cuanto a examinadores y muestra poblacional y por lo tanto se establece una regularidad de asociación más concluyente.

\section{POBLACION Y METODO}

La muestra que ha generado las estimaciones de asociación entre rendimiento escolar y neurológico ha sido descrita ${ }^{1-2-3-4}$. La muestra presente corresponde a escolares que cursan el segundo, tercero y cuarto año de Enseñanza Básica y que pertenecen a las escuelas: San Benildo, D.N. 15., Básica 34, Básica EN 48, $N^{\circ} 10$. La muestra previa comprendió casos indices de tercer afto y de segundo (repitertes un affo), cuyas edades fluctuaron entre 8 y 9 años. Consideramos, en este caso, que es más importante la edad cronológica que la edad escolar, ya que si existe un nexo causal entre ambos rendimientos, el grado escolar que el alumno tiene es irrelevante, provisto que sean incluidos números suficientes de individuos en las diversas categorías de rendimientos. Fijamos el intervalo etario entre 8.7 y 9.5 años.

Los examinadores fueron Internos de la Carreta de Medicina a quienes se preparó con un adiestramiento especial para tomar las pruebas neurológicas y de lecto-escritura. Estas pruebas están descritas en los trabajos previos. De las pruebas neurológicas elegimos entre las más discriminativas: 1) Imitar con las manos la posición de los dedos del examinador; II) Igual a I, sin ayuda visual; III) Pronunciación de tres trabalenguas; IV) Estructura del lenguaje en dicción tequerida; $y \mathrm{~V}$ ) Precisión en los movimientos secuenciales de los dedos. De entre las dudosamente discriminativas se eligieron: VI) Postura de tronco en posición firme; VII) Capacidad de aislar movimientos braquiales de los cervicales. De entre las pruebas no discriminativas se seleccionaron: VIII) Perseveración de los movimientos inducidos de braceo; IX) Capacidad de mantener por 15 segundos la convergencia ocular y X) Control de sincinecias faciales al cerrar y abrir la boca. El rendimiento escolar es catalo. gado como en el trabajo anterior en ANORMAL. (A), SUBNORMAL (SN), y NORMAL (N). El rendimiento en la ejecución de las pruebas neurológicas es catalogado de acuerdo a su adecuación en $\mathrm{J} 1, \mathrm{~J} 2, \mathrm{~J} 3$ y $\mathrm{J} 4$ siendo $\mathrm{J} 1$ el más deficientemente ejecutado.

\section{RESULTADOS}

El rendimiento comparativo de las pruebas de lecto-escritura y neurológicas en varones y mujeres está descrito en las tablas 1 y 2 respectivamente.
Tabla 1.

Rendimiento en Lecto-Escritura según sexo $y$ curso

\begin{tabular}{|c|c|c|c|c|c|c|c|c|}
\hline \multirow[b]{2}{*}{ Curso } & \multicolumn{4}{|c|}{ Mujeres } & \multicolumn{4}{|c|}{ Varones } \\
\hline & A & SN & $\mathrm{N}$ & Tot. & A & $S N$ & $N$ & Tot \\
\hline$o^{\text {No }}$ & 10 & 6 & 4 & 20 & 5 & 8 & 3 & 16 \\
\hline$\%$ & 50.00 & 30.00 & 20.00 & & 31.25 & 50.00 & 18.37 & \\
\hline No & 7 & 27 & 67 & 101 & 4 & 22 & 41 & 67 \\
\hline$\%$ & 6.93 & 26.73 & 66.34 & & 5.97 & 32.84 & 61.19 & \\
\hline $\mathrm{N}^{\mathrm{e}}$ & 0 & 12 & $\$ 1$ & 63 & 2 & 16 & 58 & 76 \\
\hline$\%$ & 0.00 & 19.05 & 80.95 & & 2.63 & 21.05 & 76.32 & \\
\hline No & 17 & 45 & 122 & 184 & 11 & 46 & 102 & 159 \\
\hline$\%$ & 9.24 & 24.46 & 66.30 & & 6.92 & 28.93 & 64.15 & \\
\hline & Poro & ntaj & Icula & os $e$ & $\mathrm{el}$ tr & jo & & \\
\hline & 15.82 & 6.5 & 77.55 & & 13.6 & 27.4 & 58.91 & \\
\hline
\end{tabular}

Tabla 2.

Rendimiento en pruebas neurológicas según sexo

\begin{tabular}{|c|c|c|c|c|c|c|c|c|c|c|}
\hline \multirow{2}{*}{\multicolumn{2}{|c|}{ Prueba }} & \multicolumn{5}{|c|}{$\begin{array}{l}\text { MLUERES } \\
\text { Readimituros }\end{array}$} & \multicolumn{4}{|c|}{$\begin{array}{l}\text { VARGNES } \\
\text { Rasideaver:to }\end{array}$} \\
\hline & & 11 & 12 & 13 & Ja & 101 & J: & 12 & 13 & 34 \\
\hline \multirow{2}{*}{ 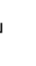 } & $n$ & $\nabla$ & 3 & $\because i$ & 100 & 185 & 0 & 15 & $6^{7}$ & BA \\
\hline & 4 & 20 & 412 & $\$ 1.62$ & 54015 & & 000 & 9.04 & 40.36 & soted \\
\hline \multirow{2}{*}{ II } & $\infty$ & 0 & 14 & 81 & เ8 & & d & 28 & $B$ & 6 \\
\hline & 3 & (I) & $75:$ & $\$ 4.86$ & $173^{4}$ & & $0 .(k)$ & . 0.3. & 50.60 & $38: 8$ \\
\hline \multirow{2}{*}{ III } & $v v$ & 0 & 1 & 1] & 150 & & 0 & 2 & 31 & 133 \\
\hline & $*$ & in & $10 \mathrm{hH}$ & 1784 & 9108 & & 0.00 & 1.2] & 18.67 & 8012 \\
\hline \multirow{2}{*}{ w } & yo & o & $n$ & 20 & 162 & & 0 & 3 & 11 & 155 \\
\hline & 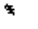 & ¿..ง & 0.00 & 12.43 & ด7.5 & & $D \mathrm{JC}$ & 0.00 & b.61 & B \\
\hline \multirow[t]{2}{*}{1} & w & D & 3 & 66 & 116 & 1 & $a$ & ;E & 90 & \\
\hline & $\phi$ & |] & 162 & $35.6 \mathrm{~F}$ & $8: 90$ & & Dso & 0.00 & 15.8 & $: 4.22$ \\
\hline \multirow[t]{2}{*}{$v_{1}$} & No & $\mathfrak{o}$ & 0 & 24 & $|s|$ & & 0 & 1 & 24 & $|4|$ \\
\hline & 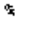 & 000 & 000 & 1243 & $8>09$ & บ 00 & 064 & $1 \Delta A \hat{H}$ & 14.04 & \\
\hline \multirow{2}{*}{ 611 } & N" & J & 1 & BU & $12:$ & a & 2 & 45 & 114 & \\
\hline & $*$ & 161 & $0 \leq 4$ & $y=26$ & 6534 & & $\theta \cos$ & 121 & 27.11 & 769 \\
\hline \multirow{2}{*}{ ?aI } & $\therefore:$ & 1 & 9 & ft & 110 & & 0 & 3 & $\because$ & 102 \\
\hline & 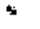 & n.ses & $+\mathrm{bi}$ & $34=9$ & 5978 & & 000 & 了 8t & 3313 & 65.06 \\
\hline \multirow{2}{*}{ JX } & $\mathrm{si}$ & 1 & .6 & $s$ & 134 & & 2 & 17 & iE & 110 \\
\hline & $\Rightarrow$ & 1134 & B 65 & $183 \mathrm{y}$ & 72.45 & & 1 on & 3634 & :68: & 71.89 \\
\hline \multirow{2}{*}{$\mathrm{x}$} & $N$ & 0 & $\mathfrak{o}$ & 59 & 126 & & 0 & 2 & is & s9 \\
\hline & 4 & 000 & v $6 r$ & 3159 & 68 it & & $\dot{\mathrm{u} .00}$ & 120 & 45.18 & 53.62 \\
\hline
\end{tabular}

Se advierte un porcentaje superior de varones en el conjunto de las categorías Anormal $y$ Subnormal, más notorio en la Subnormal, pero estas diferencias no son estad ísticamente significativas. El rendimiento en la ejecurción de pruebas neurológicas es porcentualmente superior en las mujeres en: I, II, III, V, VI, LX y X e inferior en las otras pruebas. Esta diferencia es significativa sólo en la prueba $X(P<0.01)$.

$\mathrm{La}$ verificación de la discriminación que las pruebas revelaron tener en el estudio previo es presentada en lá tabla 3; aqui sólo daremos los resultados numéricos de las pruebas neurológicas II, III, VIII, LX y X para simplificar la presentación. 
Tabla 3.

Distribución de los escolares según rendimiento en Lecto-Escritura (A, SN y N) y pruebas neurológicas (J1, J2, J3 y J4). Puuebas II, IJI, VIU, IX y X

\section{MUJERES}

VARONES

II copia de movimientos digitales sin ver

$\begin{array}{lrrrrrr} & \text { A } & \text { SN } & \text { N } & \text { A } & \text { SN } & \text { N } \\ \text { J2* } & 3 & 7 & 4 & 3 & 4 & 11 \\ \text { J3 } & 10 & 19 & 54 & 8 & 27 & 49 \\ \text { J4 } & 4 & 19 & 65 & 2 & 15 & 47\end{array}$

III pronunciación de tres trabalenguas

$\begin{array}{rrrrrrr}\text { J2 } & 2 & 0 & 0 & 1 & 1 & 0 \\ \text { J3 } & 8 & 14 & 11 & 4 & 12 & 15 \\ \text { J4 } & 7 & 31 & 112 & 8 & 33 & 92\end{array}$

VIII perseveración en movimientos inducidos de braceo

$\begin{array}{rrrrrrr}\text { J1 } & 0 & 0 & 1 & 0 & 0 & 0 \\ \text { J2 } & 2 & 5 & 2 & 2 & 0 & 1 \\ \text { J3 } & 9 & 19 & 36 & 5 & 21 & 29 \\ \text { J4 } & 6 & 20 & 84 & 6 & 25 & 77\end{array}$

IX mantención de convergencia ocular por 15 segundos

$\begin{array}{rrrrrrr}J 1 & 0 & 1 & 0 & 0 & 0 & 2 \\ J 2 & 4 & 4 & 8 & 1 & 5 & 11 \\ J 3 & 2 & 9 & 23 & 3 & 6 & 19 \\ \text { J4 } & 11 & 31 & 92 & 9 & 35 & 75\end{array}$

$X$ sincinecias faciales excesivas

$\begin{array}{rrrrrrr}\text { J2 } & 0 & 0 & 0 & 0 & 1 & 1 \\ \text { J3 } & 8 & 15 & 36 & 9 & 15 & 51 \\ \text { J4 } & 9 & 30 & 87 & 4 & 30 & 55\end{array}$

* Se omite $J 1$ cuando no hubo individuos con ese rendimiento neurológico.

De los datos ordenados cono en esta tabla se puede estudiar la discriminación de la pruebas mediante el cálculo de los valores " $t$ " $y$ de $X^{2}$; no se trata de una prueba de significación estadística, aunque puede interpretarse en esta forma, sino más bien de un sistema de ordenación del valor discriminatorio de cada prueba. Este sistema está garantizado ya que los números e individuos son los mismos para cada prueba. La discriminación se estudia entre las filas $\mathrm{J} 3$ y $\mathrm{J} 4$ (rendimiento neurológico casi correcto y correcto) por el valor de $\mathrm{X}^{2}$ con dos grados de libertad y entre las columnas $A$ y $\mathrm{N}$ (rendimiento en lecto-escritura anormal y normal) por el valor de " $t$ ". El primer análisis es de discriminación fina, y pretende dilucidar si hay un componente mayor de lecto-escritores subnomnales y anormales entre los individuos que rinden el examen neurológíco en forma casi correcta que entre los que lo rinden correctamente. El segundo análisis pretende encontsar un mayor componente de pruebas neurológicas alteradas entre los lectoescritores anormales que en los normales. Es este segundo análisis el que más se aproxima al del trabajo ya publicado y es, por lo tanto el que permite mejor la verificación. La tabla 4 presenta los valores de $\mathrm{X}^{2}$ y $\mathrm{t}$ para cada prueba $y$ sexo, se agrega además el lugar que le correspondió de acuerdo a su capacidad discriminatoria en el trabajo anterior. El valor de $X^{2}$ es significativo al $5 \%$ por encima de 5.9 y el de t por encima de 1.7 .

Tabla 4.

Valores de $\mathrm{X}^{2}$, t $\mathrm{y}$ lugar en la clasificación anterior de las 10 pruebas neurológicas según sexo

\begin{tabular}{lrrrrr} 
Prueba & \multicolumn{2}{c}{ MUJERES } & Lugar & \multicolumn{2}{c}{ VARONES } \\
& \multicolumn{1}{c}{$\mathrm{X}^{2}$} & $\mathrm{t}$ & & \multicolumn{1}{c}{$\mathrm{X}^{2}$} & $\mathrm{t}$ \\
I & 5.3 & 1.8 & $40^{\circ}$ & 9.6 & 3.1 \\
II & 3.4 & 2.6 & $1^{\circ}$ & 4.4 & 2.2 \\
III & 24.7 & 3.7 & $5^{\circ}$ & 5.1 & 1.7 \\
IV & 33.3 & 3.8 & $20^{\circ}$ & 0.5 & 0.3 \\
V & 6.2 & 2.2 & $30^{\circ}$ & 3.6 & 1.2 \\
VI & 1.9 & 1.0 & $6^{\circ}$ & 5.4 & 0.7 \\
VII & 7.7 & 1.9 & $70^{\circ}$ & 10.2 & 3.0 \\
VIII & 8.2 & 2.5 & $20^{\circ}$ & 2.5 & 1.9 \\
IX & 0.3 & 1.2 & $24^{\circ}$ & 0.9 & -0.3 \\
X & 2.2 & 1.4 & $25^{\circ}$ & 0.5 & -0.3
\end{tabular}

El resultado más evidente es que las pruebas IX y X no tuvieron ningún grado de asociación con la capacidad de aprendizaje de la lecto-escritura en ambas experiencias. Otros dos resultados son contradictorios: la prueba Vl discriminaba en el primer estudio, no tiene ninguna discriminación en el actual y la prueba VIII que no era discriminativa lo es en la muestra presente. En el resto se cumple lo esperado con algunas discordancias si se considera el factor sexo. Las pruebas I y Il que ocupaban, en la primera experiencia, el $4^{\circ}$ y $1^{\circ}$ lugar en la escala de discriminación ocupan el $7^{\circ}$ y $3^{\circ}$ lugar, según la prueba $t$ en las mujeres y el $1^{\circ}$ y $3^{\circ}$ lugar en los varones, de manera que sólo en varones se establece significación estadística de correlación en estas dos priebas por el cálculo de $\mathrm{X}^{2}$. Ambas se reflejan disminuidas en su capacidad de discriminación en relación al trabajo anterior. Las pruebas III y IV son las más discriminativas tanto en $t$ como en $\mathrm{X}^{2}$ en las mujeres, en cambio IV no presenta discriminación en varones y III es dudosamente discriminativa. La prueba VII es ahora claramente discriminativa.

El cumplimiento de las predicciones que hicimos sobre las proporciones esperadas de individuos con un determinado rendimiento en lecto-escritura según rendimiento neurológico puede evaluarse comparando las matrices semejantes a las de la tabla 3 de ambos trabajos. Aquí, sólo compararemos las pruebas II, III, VIII y IX.

Las diferencias entre las proporciones de ambos estudios no impiden encontrar un patrón genera] caracterizado, por ejemplo, por un aumento de las 
probabilidades de ser buen lector cuando la ejecución de la prueba neurológica ha sido satisfactoria en las pruebas discriminativas; patrón que no se observa en las pruebas no discriminativas.

Tabla 5.

Comparación de probabilidades directas con probabilidades calculadas por la inversión bay esiana

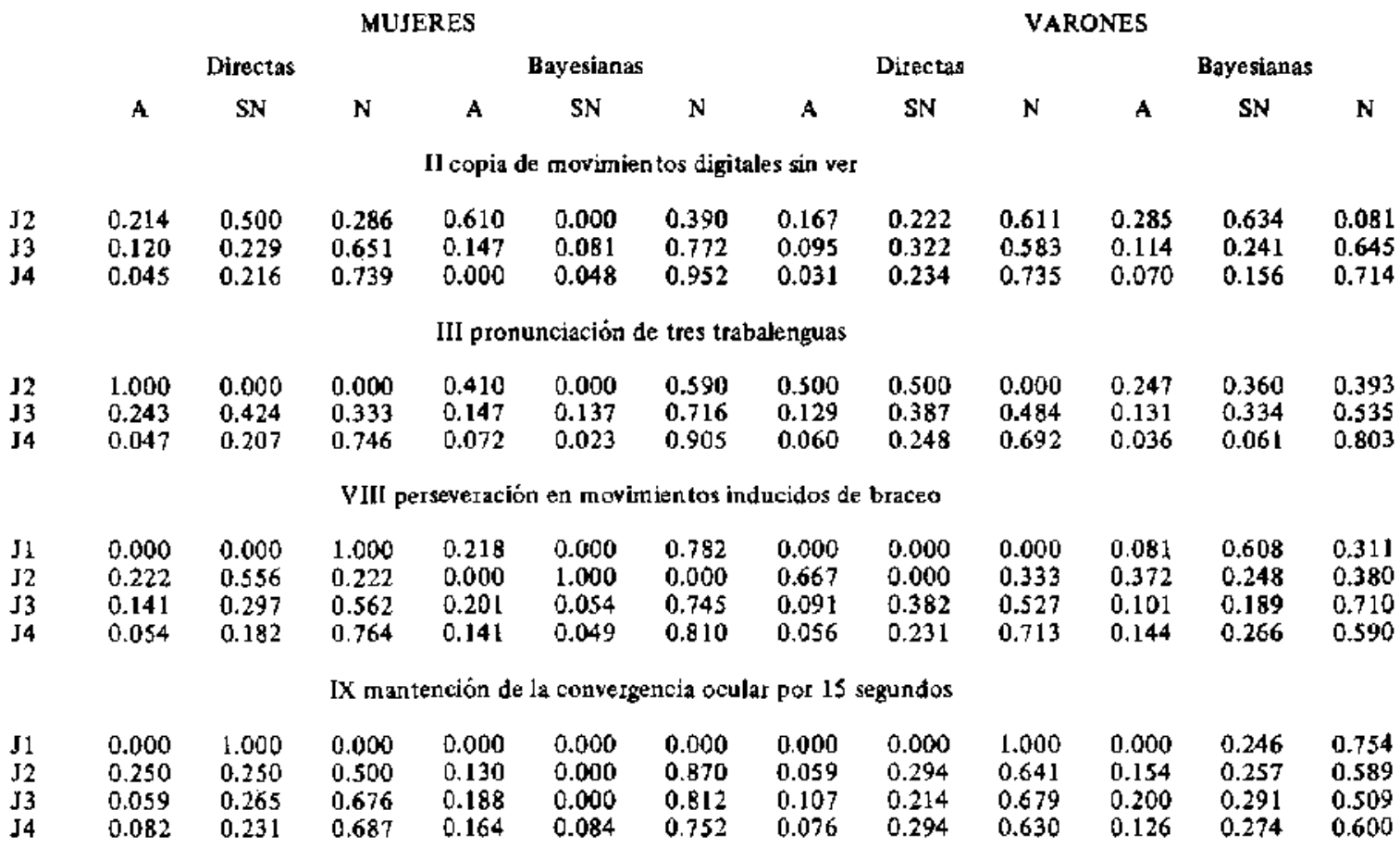

\section{DISCUSION}

Los trabajos comparados tienen casuísticas y métodos distintos entre sí y de allí la explicación de las incongruencias entre ellos. El primero tomó niños con rendimiento escolar anormal con sus respectivos controles de una muestra aleatoria en su origen, pero que, después de 3 años de segujmiento es dificil que se encuentre en su estado primitivo de aleatoriedad. Por lo tanto, corresponde a una búsqueda intencionada y sistemática de niños con rendimiento escolar bajo, lo que explica la mayor proporción de categorías $\mathrm{A}$ y $\mathrm{SN}$ que de la $\mathrm{N}$ en dicha muestra. $\mathrm{El}$ segundo estudio tomó niffos de escuelas seleccionadas únicamente por su ubicación geográfica, tratando que correspondiesen a poblaciones de nivel medio socioeconómico. Se consideraron sólo sujetos cuyas edades estuviesen entre 8.7 y 9.5 años, sin considerar distribución por grados educacionales, a diferencia de la primera muestra, que incluyó solamente niños con 5 semestres completos de educación primaria. En cambio en este estudio se incluyen nifros de $4^{\circ}$ Básico que necesariamente deben tener un mejor rendimiento escolar, lo que reduce los casos infor- mativos de la interacción entre lecto-escritura y ejecución de pruebas neurológicas.

La gran variación entre ambos estudios consistió en el aumento considerable de mujeres clasificadas como subnormales lecto-escritoras. Según los presentes resultados, el dimorfismo sexual sería menor que el del trabajo anterior. El mejor rendimiento en ambos tipos de pruebas por parte de las mujeres encontrado en el primer trabajo se confirma aquí sólo parcial y más débilmente.

Lo más importante es que los resultados de esta investigación parecen confirmar nuestra hipótesis emitida en la publicación anterior de que es posible encontrar pruebas de orden neuropsicológico que tienen un valor de signo asociado al rendimiento escolar, y que cuanto más compleja desde el punto de vista fisiológico es la prueba, más relaciones tiende a tener con el rendimiento en lectoescritura. Las pruebas simples, más próximas a los reflejos primarios no mostraron relación con las pruebas en lecto-escritura.

No se pretende que estas pruebas, en el estado actual en que las hemos aplicado, sean herramientas predictivas del rendimiento escolar, pero, a través de estos resultados se abre un camino para 
perfeccionarlas, to cual podrá desentrañar el mecanismo de esta relación reiteradamente puesta en evidencia. Así, nos podemos preguntar: ¿Cuáles son aquellos procesos y funciones neurológicas más necesarios para un buen rendiniento escolar? . Es licito pensar que aquellos que signifiquen una sutil y compleja integración intersensorial estarán más involucrados con los procesos de aprendizaje que las sincinecias faciales o la capacidad de convergencia ocular de configuración neurológica, supuestamente más simples en su estructura funcional.

Estos resultados justifican plantearse el problema de la adecuación de los cursos y programas escolares más bien a la edad de desarrollo neurológico que a la del cronológico y la necesidad de una educación diferenciada desde el comienzo.

\section{RESUMEN}

En trabajos previos encontramos que algunas pruebas neurológicas podían discriminar grupos de escolares con dificultad en el proceso de lectoescritura. Esos estudios contenían varias instancias de sesgos de pesquisa, por lo que realizamos el presente trabajo para verificar lo encontrado previamente. Elegimos pruebas neurológicas que resultaron muy discriminativas ( 5 de ellas), pruebas neurológicas que resultaron próximas a la discriminación ${ }^{2}$ y pruebas que resultaron no discriminativas ${ }^{3}$. Se confirmó, en general, lo encontrado en los trabajos previos, con variaciones en el poder de discriminación de las pruebas. Aunque la proporción de niffas con rendimiento deficiente en lecto escritura es inferior a lo encontrado en nifios en el estudio actual, este dimorfismo está muy reducido en relación al trabajo previo.

Financiado por Proyecto M797-801 del Servicio de Desarrollo Científico, Artístico y de Cooperación Inter. nacional de la Universidad de Chile; por el Convenio Unidad de Genética Humana-CECOM; y por los recursos ordinarios de la Universidad de Chile.

\section{REFERENCIAS}

1 Manterola, A.; Avendaño, A.; Volenzuela, C.; Figueron, L.: Pacheco, L.: Kartzow, M.: y Fernindez, M. Estudio sobre prevalencia y características biológicas, socioculturales y psicológicas de niños con insuficiente rendimiento escolar. I Parte: Diseño de la investigación. Rev. Chil. Pediat. 52, 1, 90-98, 1981.

${ }^{2}$ Manterola, A.; Avendaño, A.; Valenzuela, C.Y. Figuerod, L.; Pacheco, L.; Aviln, S.: Fernóndez, M.; Escuedero, $M$. Estudio sobre prevalencia y caracteristicas biológicas, socioculturales y psicológicas de nifios con insuficiente rendiniento escolar. Il Parte: Aspectos socioculturales y biológicos. Rev. Chil. Pediat. 52, 2, 155-163, 1981 .

3 Manterola, A.; Avendario, A.: Valenzuela, C.Y.; Figueroa, L.; Pacheco, L.; Kartzow, M.; Ferníndez, M.; Morales, $I$. Estudio sobre prevalencin y caracteristicas biológicas socioculturales y psicológicas de niños con insuffeiente rendimiento escolar. III Parte: análisis de algunos resultados de exámenes neurológicos y psicológicos. Rev. Chil. Pediat. 52, 3, 250-262, 1981.

4 Vatenzuela, C.Y.; Morales, 1. Estudio Bayesiano del rendimiento escolar según diez pruebas neurológicas. Rev. Chil. Pediat. 52, 6, 486-490, 1981. 